\title{
A NOTE ON WALSH-FOURIER SERIES
}

\author{
WO-SANG YOUNG
}

\begin{abstract}
It is shown that the double sequence $\left\{\lambda_{m n}\right\}$ with $\lambda_{m n}=1$ if $n<m$ and 0 otherwise is an $L^{p}$ multiplier for the Walsh system in two dimensions only if $p=2$. This result is then used to show that the one-dimensional trigonometric system and the Walsh system are nonequivalent bases of the Banach space $L^{p}[0,1]$, and hence have different $L^{p}$ multipliers, $1<p<\infty, p \neq 2$.
\end{abstract}

1. Let $\left\{\lambda_{m n}\right\}_{-\infty<m, n<\infty}$ be the double sequence defined by $\lambda_{m n}=1$ if $n \leqslant m$ and 0 otherwise. For

$$
f \sim \sum_{m, n=-\infty}^{\infty} a_{m n} e^{2 \pi i(m x+n y)} \in L^{p}([0,1] \times[0,1]),
$$

let

$$
T_{1} f \sim \sum_{m, n=-\infty}^{\infty} a_{m n} \lambda_{m n} e^{2 \pi i(m x+n y)} .
$$

It is well known that $T_{1}$ is bounded on $L^{p}([0,1] \times[0,1]), 1<p<\infty$. This is a consequence of the one-dimensional result of $\mathrm{M}$. Riesz for the conjugate function [6, I, p. 253]. The $L^{p}$ boundedness of $T_{1}$ was used, for example, in C. Fefferman's proof of the almost everywhere convergence of double Fourier series [1].

We now turn our attention to the Walsh system $\left\{w_{n}\right\}$. For

$$
f \sim \sum_{m, n=0}^{\infty} a_{m n} w_{m}(x) w_{n}(y) \in L^{p}([0,1] \times[0,1]),
$$

consider the corresponding operator $T_{2}$ defined by

$$
T_{2} f \sim \sum_{m, n=0}^{\infty} a_{m n} \lambda_{m n} w_{m}(x) w_{n}(y) .
$$

Because of the great similarity between the Walsh system and the trigonometric system, one would expect $T_{2}$ to be bounded on $L^{p}([0,1] \times[0,1])$, $1<p<\infty$. However, this is not the case. In $\S 2$ we will show that $T_{2}$ is not bounded on $L^{p}$ except for $p=2$. This result is then used in $\S 3$ to give a negative answer to a question of P. Enflo: Are the trigonometric system and the Walsh system equivalent bases of the Banach space $L^{p}[0,1], 1<p<\infty$, $p \neq 2$ ? Finally in $\S 4$ we will deduce from the nonequivalence that the

Received by the editors July 25, 1975.

AMS (MOS) subject classifications (1970). Primary 42A18, 42A56, 42A92; Secondary 46B15, 46E30. 
one-dimensional Walsh system and trigonometric system have different $L^{p}$ multipliers, $1<p<\infty, p \neq 2$.

2. Let $\left\{r_{n}\right\}_{n>0}$ denote the Rademacher functions and $\left\{w_{n}\right\}_{n \geqslant 0}$ the Walsh functions defined on $I=[0,1]$. For any two real numbers with dyadic expansions $a=\sum_{j=-\infty}^{\infty} \alpha_{j} 2^{j}, b=\sum_{j=-\infty}^{\infty} \beta_{j} 2^{j}, \alpha_{j}, \beta_{j}=0$ or 1 , let $a \dot{+} b=$ $\sum_{j=-\infty}^{\infty}\left|\alpha_{j}-\beta_{j}\right| 2^{j}$. It is understood that we use the finite representation in the case of a dyadic rational. Basic properties of the Walsh functions can be found in [2].

THEOREM 1. $T_{2}$ is bounded on $L^{p}\left(I^{2}\right)$ if and only if $p=2$.

Proof. The case $p=2$ is trivial by Parseval's formula. It is sufficient to show that $T_{2}$ is not bounded on $L^{p}$ for $p<2$, for then the theorem will follow by a duality argument.

Instead of dealing with $T_{2}$ we consider an equivalent operator $T_{2}^{\prime}$ defined on $L^{p}\left(I^{2}\right)$ as follows. Let $A=\{(m, n): 0 \leqslant n \leqslant m \dot{+}, m=0,1, \ldots\}$. For $f \sim \sum_{m, n=0}^{\infty} a_{m n} w_{m}(x) w_{n}(y)$, let $T_{2}^{\prime} f \sim \sum_{(m, n) \in A} a_{m n} w_{m}(x) w_{n}(y)$. Suppose $g$ is the function on $I^{2}$ defined by $g(x, y)=f(x, x+y)$. Since $w_{n}(x+y)=$ $w_{n}(x) w_{n}(y)$ and $w_{m+n}=w_{m} w_{n}$, we have $T_{2}^{\prime} g(x, y)=T_{2} f(x, x \dot{+} y)$. Therefore the boundedness of $T_{2}$ is equivalent to that of $T_{2}^{\prime}$.

We will define a sequence of functions $\left\{f_{k}\right\}$ on $I^{2}$ and show that

$$
\left\|T_{2}^{\prime} f_{k}\right\|_{p} /\left\|f_{k}\right\|_{p} \rightarrow \infty \quad \text { as } k \rightarrow \infty
$$

Let

where

$$
f_{k}=\sum_{l=1}^{k} f_{k l}
$$

$$
f_{k l}(x, y)=2^{-k} r_{l-1}(x) \prod_{j=0}^{k-1}\left(1+r_{j}(y)\right)
$$

We first note that

$$
\left\|f_{k l}\right\|_{p}^{p}=\int\left|2^{-k} \prod_{j=0}^{k-1}\left(1+r_{j}(y)\right)\right|^{p} d y=2^{-k p} \int_{\left[0,2^{-k}\right]} 2^{k p} d y=2^{-k}
$$

Moreover, since $\int\left|\sum_{l=0}^{k-1} r_{l}(x)\right|^{p} d x \leqslant C_{p}^{p} k^{p / 2}$ by Khintchin's inequality [6, I, p. 213], we have

(2) $\left\|f_{k}\right\|_{p}^{p}=\int\left|\sum_{l=0}^{k-1} r_{l}(x)\right|^{p} d x \int\left|2^{-k} \prod_{j=0}^{k-1}\left(1+r_{j}(y)\right)\right|^{p} d y \leqslant C_{p}^{p} k^{p / 2} 2^{-k}$,

where $C_{p}$ denotes a constant depending only on $p$.

On the other hand, for $1 \leqslant l \leqslant k$,

$$
\begin{aligned}
T_{2}^{\prime} f_{k l}(x, y)= & 2^{-k} \sum_{n<m+n ; m} \int r_{l-1}(s) w_{m}(x) d s \\
& \cdot \int \prod_{j=0}^{k-1}\left(1+r_{j}(t)\right) w_{n}(t) d t w_{m}(x) w_{n}(y) .
\end{aligned}
$$


Now

$$
\int r_{l-1}(s) w_{m}(s) d x=1 \text { if } m=2^{l-1}
$$

and 0 otherwise. Also

Therefore

$$
\begin{aligned}
\int \prod_{j=0}^{k-1}\left(1+r_{j}(t)\right) w_{n}(t) d t & =1 \quad \text { if } n<2^{k} \\
& =0 \text { if } n \geqslant 2^{k} .
\end{aligned}
$$

$$
T_{2}^{\prime} f_{k l}(x, y)=2^{-k} \sum_{n<2^{l-1}+n ; n<2^{k}} w_{n}(y) w_{2^{\prime-1}}(x) .
$$

Let $n=\sum_{j=0}^{\infty} \varepsilon_{j} 2^{j}$ with $\varepsilon_{j}=0$ or 1 . We observe that $n \leqslant 2^{l-1} \dot{+} n$ if and only if $\varepsilon_{l-1}=0$. Therefore

$$
\begin{aligned}
& \sum_{n<2^{l-1}+n ; n<2^{k}} w_{n}(y)=\prod_{0<j<k ; j \neq l-1}\left(1+r_{j}(y)\right) \\
& =\frac{1}{2}\left[\prod_{0<j<k}\left(1+r_{j}(y)\right)+\prod_{0<j<k}\left(1+r_{j}\left(y+2^{-l}\right)\right)\right] .
\end{aligned}
$$

From (3) and (4), we have

$$
T_{2}^{\prime} f_{k l}(x, y)=\frac{1}{2}\left[f_{k l}(x, y)+f_{k l}\left(x, y+2^{-l}\right)\right] \text {, }
$$

and hence

$$
T_{2}^{\prime} f_{k}(x, y)=\frac{1}{2} f_{k}(x, y)+\frac{1}{2} \sum_{l=1}^{k} f_{k l}\left(x, y+2^{-l}\right) .
$$

Since $f_{k}(x, y)$ and $f_{k l}\left(x, y+2^{-l}\right), l=1, \ldots, k$, have mutually disjoint supports, it follows from (1) that

$$
\left\|T_{2}^{\prime} f_{k}\right\|_{p}^{p} \geqslant 2^{-p} \sum_{l=1}^{k}\left\|f_{k l}\right\|_{p}^{p}=2^{-p} k 2^{-k} .
$$

Combining (2) and (5), we obtain, for $p<2$,

$$
\left\|T_{2}^{\prime} f_{k}\right\|_{p} /\left\|f_{k}\right\|_{p} \geqslant 2^{-1} C_{p}^{-1} k^{(1 / p-1 / 2)} \rightarrow \infty \quad \text { as } k \rightarrow \infty .
$$

This completes the proof of Theorem 1.

3. It is known that $\{\cos \pi n x\}$ and $\left\{w_{n}\right\}$ are bases of the Banach space $L^{p}(I), 1<p<\infty$. (See [6, I, p. 266] and [4].) We say that the sequences $\left\{u_{n}\right\},\left\{v_{n}\right\}$ of a Banach space are equivalent if for every sequence of numbers $\left\{a_{n}\right\}, \sum_{n=0}^{\infty} a_{n} u_{n}$ converges if and only if $\sum_{n=0}^{\infty} a_{n} v_{n}$ converges. R. Askey, S. Wainger and J. E. Gilbert showed that $\{\cos \pi n x\}$ and certain classical orthonormal sequences are equivalent in $L^{p}(I), 1<p<\infty$. (See [3].) We have the following

TheOREM 2. Let $1<p<\infty .\{\cos \pi n x\}$ and $\left\{w_{n}\right\}$ are equivalent bases of $L^{p}(I)$ if and only if $p=2$.

Proof. Again the case $p=2$ is trivial by Parseval's formula. Suppose they 
were equivalent in $L^{p}(I), p \neq 2$. From this it would follow that $\left\{e^{2 \pi i n x}\right\}_{n \geqslant 0}$ and $\left\{w_{n}\right\}$ are also equivalent in $L^{p}(I)$. (See [6, I, p. 253].) By the BanachSteinhaus theorem, there exist constants $C_{p}, C_{p}^{\prime}>0$ such that for any sequence of numbers $\left\{a_{n}\right\}$,

$$
C_{p}^{-1}\left\|\sum_{n=0}^{N} a_{n} e^{2 \pi i n x}\right\|_{p} \leqslant\left\|\sum_{n=0}^{N} a_{n} w_{n}\right\|_{p} \leqslant C_{p}^{\prime}\left\|\sum_{n=0}^{N} a_{n} e^{2 \pi i n x}\right\|_{p}, \quad N \geqslant 0 .
$$

(See $\left[5\right.$, p. 70].) Let $\left\{a_{m n}\right\}$ be any double sequence of numbers. Applying (6) first to the $x$-variable and then to the $y$-variable, we obtain

$$
\begin{aligned}
C_{p}^{-2}\left\|\sum_{m, n=0}^{N} a_{m n} e^{2 \pi i(m x+n y)}\right\|_{p} & \leqslant\left\|\sum_{m, n=0}^{N} a_{m n} w_{m}(x) w_{n}(y)\right\|_{p} \\
& \leqslant C_{p}^{\prime 2}\left\|\sum_{m, n=0}^{N} a_{m n} e^{2 \pi i(m x+n y)}\right\|_{p}, \quad N \geqslant 0 .
\end{aligned}
$$

Now, it follows from the corresponding one-dimensional result that for any function in $L^{p}\left(I^{2}\right)$, the square partial sums of both its trigonometric Fourier series and Walsh-Fourier series converge in $L^{p}\left(I^{2}\right)$. Therefore (7) implies the following: for any sequence of numbers $\left\{a_{m n}\right\}, f \sim \sum_{m, n=0}^{\infty} a_{m n} e^{2 \pi i(m x+n y)}$ for some $f \in L^{p}\left(I^{2}\right)$ if and only if $g \sim \sum_{m, n=0}^{\infty} a_{m n} w_{m}(x) w_{n}(y)$ for some $g \in$ $L^{p}\left(I^{2}\right)$.

Suppose $g \in L^{p}\left(I^{2}\right)$ with $g \sim \sum_{m, n=0}^{\infty} a_{m n} w_{m}(x) w_{n}(y)$. Then $f$ $\sim \sum_{m, n=0}^{\infty} a_{m n} e^{2 \pi i(m x+n y)} \in L^{p}\left(I^{2}\right)$. Hence

which implies

$$
T_{1} f \sim \sum_{m, n=0}^{\infty} a_{m n} \lambda_{m n} e^{2 \pi i(m x+n y)} \in L^{p}\left(I^{2}\right)
$$

$$
T_{2} g \sim \sum_{m, n=0}^{\infty} a_{m n} \lambda_{m n} w_{m}(x) w_{n}(y) \in L^{p}\left(I^{2}\right) .
$$

Therefore $T_{2}$ maps $L^{p}\left(I^{2}\right)$ into $L^{p}\left(I^{2}\right)$. By the closed graph theorem, $T_{2}$ is bounded on $L^{p}\left(I^{2}\right)$, contradicting Theorem 1. This proves Theorem 2 .

4. Let $\left\{u_{n}\right\}$ be one of the sequences $\left\{w_{n}\right\},\{\cos \pi n x\}$ or $\left\{e^{2 \pi i n x}\right\}_{n \geqslant 0}$. $M\left(L^{p},\left\{u_{n}\right\}\right)$ denotes the collection of all sequences $\left\{\lambda_{n}\right\}$ such that $f \sim$ $\sum_{n=0}^{\infty} a_{n} u_{n} \in L^{p}(I)$ implies $g \sim \sum_{n=0}^{\infty} \lambda_{n} a_{n} u_{n} \in L^{p}(I)$. We will deduce from Theorem 2 the following

TheOREM 3. $M\left(L^{p},\{\cos \pi n x\}\right) \neq M\left(L^{p},\left\{w_{n}\right\}\right), 1<p<\infty, p \neq 2$.

We note that in general two nonequivalent bases of $L^{p}(I)$ may have the same multipliers. See, for example, [5, p. 484 and p. 546].

Proof. Suppose they were equal. Since

$$
M\left(L^{p},\{\cos \pi n x\}\right)=M\left(L^{p},\left\{e^{2 \pi i n x}\right\}_{n>0}\right),
$$

we have $M\left(L^{p},\left\{w_{n}\right\}\right)=M\left(L^{p},\left\{e^{2 \pi i n x}\right\}_{n \geqslant 0}\right)$. Let $\sum_{n=0}^{\infty} a_{n} e^{2 \pi i n x} \in L^{p}$. For every $t \in[0,1]$,

$$
\left\|\sum_{n=0}^{\infty} a_{n} e^{2 \pi i n t} e^{2 \pi i n x}\right\|_{p}=\left\|\sum_{n=0}^{\infty} a_{n} e^{2 \pi i n x}\right\|_{p}
$$


so $\left\{e^{2 \pi i n t}\right\} \in M\left(L^{p},\left\{e^{2 \pi i n x}\right\}\right)$, and hence belongs to $M\left(L^{p},\left\{w_{n}\right\}\right)$. We assert that, moreover, there is a constant $C_{p}$, depending only on $p$, such that for every $\sum_{n=0}^{\infty} a_{n} w_{n} \in L^{p}$,

$$
\int\left|\sum_{n=0}^{\infty} a_{n} e^{2 \pi i n t} w_{n}(x)\right|^{p} d x \leqslant C_{p}^{p} \int\left|\sum_{n=0}^{\infty} a_{n} w_{n}(x)\right|^{p} d x, \quad t \in[0,1] .
$$

We will prove (8) by contradiction. Suppose there was no such constant. Then there would exist $\left\{t_{k}\right\} \subset[0,1],\left\{a_{n}^{(k)}\right\}_{n, k \geqslant 0}$ and integers $0 \leqslant N_{0}<N_{1}$ $<\ldots$ such that

$$
\left\|\sum_{n=0}^{2^{N_{k}}-1} a_{n}^{(k)} w_{n}\right\|_{p}=1 \text { and }\left\|\sum_{n=0}^{2^{N_{k}}-1} a_{n}^{(k)} e^{2 \pi i n t_{k}} w_{n}\right\|_{p} \geqslant 2^{2 k}
$$

Observe that for $n=0,1, \ldots, 2^{N_{k}}-1, n \dot{+} 2^{N_{k}}=n+2^{N_{k}} \in\left[2^{N_{k}}, 2^{N_{k}+1}\right) \subset$ $\left[2^{N_{k}}, 2^{N_{k+1}}\right.$ ). Define a sequence $\left\{\lambda_{n}\right\}$ by

$$
\lambda_{n}= \begin{cases}0 & \text { if } 0 \leqslant n<2^{N_{0}} \\ 2^{-k} e^{2 \pi i n t_{k}} & \text { if } 2^{N_{k}} \leqslant n<2^{N_{k+1}}, k=0,1, \ldots\end{cases}
$$

Then, for $\sum_{n=0}^{\infty} a_{n} e^{2 \pi i n x} \in L^{p}$,

$$
\begin{aligned}
\left\|\sum_{n=0}^{\infty} \lambda_{n} a_{n} e^{2 \pi i n x}\right\|_{p} & \leqslant \sum_{k=0}^{\infty} 2^{-k}\left\|\sum_{n=2^{N_{k}}}^{2^{N_{k}+1-1}} a_{n} e^{2 \pi i n x}\right\|_{p} \\
& \leqslant C_{p}\left\|\sum_{n=0}^{\infty} a_{n} e^{2 \pi i n x}\right\|_{p} .
\end{aligned}
$$

(See [6, I, p. 266].) Hence $\left\{\lambda_{n}\right\} \in M\left(L^{p},\left\{e^{2 \pi i n x}\right\}\right)$. On the other hand, for $k=0,1, \ldots$,

$$
\left\|\sum_{n=0}^{2^{N_{k}}-1} a_{n}^{(k)} w_{2^{N_{k}}+n}\right\|_{p}=1
$$

whereas

$$
\left\|\sum_{n=0}^{2^{N_{k}}-1} a_{n}^{(k)} \lambda_{2^{N_{k}}+n} w_{2^{N_{k}}+n}\right\|_{p}=2^{-k}\left\|\sum_{n=0}^{2^{N_{k}}-1} a_{n}^{(k)} e^{2 \pi i n t_{k}} w_{n}\right\|_{p} \geqslant 2^{k} .
$$

By the closed graph theorem, $\left\{\lambda_{n}\right\} \notin M\left(L^{p},\left\{w_{n}\right\}\right)$, contradicting our assumption. This proves (8). Similarly we can show that there is a constant $C_{p}^{\prime}$ such that for every $\sum_{n=0}^{\infty} a_{n} e^{2 \pi i n t} \in L^{p}$,

$$
\int\left|\sum_{n=0}^{\infty} a_{n} w_{n}(x) e^{2 \pi i n t}\right|^{p} d t \leqslant C_{p}^{\prime p} \int\left|\sum_{n=0}^{\infty} a_{n} e^{2 \pi i n t}\right|^{p} d t, \quad x \in[0,1] .
$$

We will now show that (8) and (9) imply the equivalence of $\left\{w_{n}\right\}$ and $\left\{e^{2 \pi i n x}\right\}$. To see this, let $a_{1}, \ldots, a_{N}$ be any numbers. From (8), we have

$$
\iint\left|\sum_{n=0}^{N} a_{n} e^{2 \pi i n t} w_{n}(x)\right|^{p} d x d t \leqslant C_{p}^{p} \int\left|\sum_{n=0}^{N} a_{n} w_{n}(x)\right|^{p} d x .
$$


From (9), we have

$$
\int\left|\sum_{n=0}^{N} a_{n} e^{2 \pi i n t}\right|^{p} d t \leqslant C_{p}^{\prime p} \iint\left|\sum_{n=0}^{N} a_{n} w_{n}(x) e^{2 \pi i n t}\right|^{p} d t d x .
$$

Therefore $\left\|\sum_{n=0}^{N} a_{n} e^{2 \pi i n t}\right\|_{p} \leqslant C_{p} C_{p}^{\prime}\left\|\sum_{n=0}^{N} a_{n} w_{n}\right\|_{p}$. Similarly, we have

$$
\left\|\sum_{n=0}^{N} a_{n} w_{n}\right\|_{p} \leqslant C_{p} C_{p}^{\prime}\left\|\sum_{n=0}^{N} a_{n} e^{2 \pi i n t}\right\|_{p} .
$$

This shows $\left\{w_{n}\right\}$ and $\left\{e^{2 \pi i n x}\right\}_{n \geqslant 0}$ are equivalent, which contradicts Theorem 2. This completes the proof of Theorem 3 .

Acknowledgement. The author would like to thank Professor R. A. Hunt and Professor A. P. Calderón for their suggestions.

\section{REFERENCES}

1. C. Fefferman, On the convergence of multiple Fourier series, Bull. Amer. Math. Soc. 77 (1971), 744-745.

2. N. J. Fine, On the Walsh functions, Trans. Amer. Math. Soc. 65 (1949), 372-414. MR 11, 352.

3. J. E. Gilbert, Maximal theorems for some orthogonal series. I, Trans. Amer. Math. Soc. 145 (1969), 495-515. MR 40 \#6156.

4. R. E. A. C. Paley, A remarkable series of orthogonal functions. I, Proc. London Math. Soc. 34 (1932), 241-264.

5. I. Singer, Bases in Banach spaces. I, Die Grundlehren der math. Wissenschaften, Band 154, Springer-Verlag, New York and Berlin, 1970. MR 45 \#451.

6. A. Zygmund, Trigonometric series. Vols. 1, 2, Cambridge Univ. Press, New York, 1968. MR 38 \#4882.

Department of Mathematics, University of Chicago, Chicago, Illinois 60637

Current address: Department of Mathematics, Rutgers University, New Brunswick, New Jersey 08903 